\title{
THE BODY PLETHYSMOGRAPH
}

\author{
Gordon Cumming, M.B., Ph.D., A.R.I.C. \\ Formerly Lecturer in Medicine, The Department of Medicine, The Queen Elizabeth Hospital, Birmingham*
}

INVESTIGATION of the mechanical factors involved in respiration requires the measurement of a number of variables. Since all the variables are interdependent, measurements would be most valuable if they were simultaneous, but since this is not yet possible the determinations are made as closely spaced in time as possible and during one maintained pattern of respiration.

The measurements to be made include the resistance of the airways, which is given by dividing the rate of airflow from the mouth by the pressure in the alveolus. Direct measurement of alveolar pressure is not possible, but it may readily be inferred using the body plethysmograph, which has previously been described (Mead and Whittenberger, I953; DuBois, Botelho, Bedell, Marshall and Comroe, 1956).

Also to be determined are the total compliance, which is the volume of gas exhaled divided by the appropriate intrapleural pressure change, and the total thoracic gas volume.

These determinations should be possible over a wide range of respiratory frequency.

In the light of experience with the first instruments, a review of design requirements has been published (DuBois, Botelho and Comroe, 1959) which indicates that the original plethysmograph chambers probably erred towards greater rigidity than was essential, and the authors point out that lighter construction in plastic or wood is possible without prejudicing function. This type of construction might ease the problems of permitting easy patient access.

Measurements using the body plethysmograph involve the determination of dynamic pressures of a small order and this makes stringent demands on the manometer system.

The choice of pressure transducer and associated amplifiers is not critical, but if pressure records are to be free of spurious changes with frequency the apparatus as a whole should be free from gross amplitude distortion and phase-shift distortion over the measured range.

*Present address: Bellevue Chest Service, 27th Street, New York 16, U.S.A.
Amplitude distortion occurs in the systems which are under- or overdamped and measuring frequencies of the same order as the resonant frequency. It will therefore be seen that the choice of manometer system involves effecting a suitable compromise between the conflicting requirements of high sensitivity and stability on one hand, and a high natural frequency on the other.

Phase-shift may have mechanical origins, in the transducer head and its pressure line, or in the amplifier and any associated filters.

Mechanical phase-shift may be minimized by the inclusion in the pressure line of suitable damping devices in the form of a constriction, possibly variable, until the amplitude distortion is reduced to a minimum.

Phase-shift of electrical origin can also be partially compensated by mechanical damping, provided that the phase-shift angle is only a few degrees. This technique is described by Miller and Simmons (1960).

When carrier amplifiers are used in the manometer system the filter removing residual carrier may introduce considerable phase-shift, particularly when the carrier frequency is in the audible range. Since observations are made on an oscilloscope, with its extended frequency range, the unfiltered signal cannot be used. This limitation is serious in carrier amplifiers of relatively low carrier frequency.

Good stability of the pressure signal is important, especially since the pressure signal from the pneumotachygraph is integrated, where a small out-of-balance signal would have marked effect. Methods of integration vary, the commonest being the inclusion of a suitable resistance/capacitance net before a d.c. amplifier. This suffers from the disadvantage of a relatively slow response time and can be improved upon by using a d.c. àmplifier in which the R-C net is included in the feedback loop and has the added advantage of improved stability.

To shorten the time necessary for the determinations, the selection and display of the volume, flow and pressure signals in appropriate sequence should be effected by the minimum of controls. 
Controls can be reduced to a single selector by appropriate circuit design coupled with the use of electro-magnetic operation for taps. This enables determination of all parameters to be made in a few seconds.

Compliance has usually been measured as described by Mead and Whittenberger (1953), which subtracts from the pressure/volume loop a voltage proportional to flow, the slope of the resulting line being a function of compliance. A second operation, or a second circuit, in a similar way subtracts a signal proportional to volume from the pressure/flow loop, the slope of the resulting line being proportional to total resistance.

Miller and Simmons (1960) describe a simplified operation, in which the volume voltage is subtracted from the pressure/flow loop yielding the sloping resistance line. The proportion of the volume signal so subtracted is a function of total compliance.

From the resistance line is then subtracted a proportion of the flow signal which converts the sloping line into a horizontal one. This applied voltage is then a function of the pulmonary resistance. The sequence of operations is thus to close the loop with one control and to level it with a second-the readings of the two controls then yielding compliance and resistance.

This null deflection method permits a more rapid and precise determination. Both parameteêss are determined simultaneously and there is no necessity for calibration of the oscilloscope.

The mechanical behaviour of the lungs varies with the position in the total lung volume at which the determination is made, and also with the value of the airways resistance. It is therefore important that the functional residual capacity and the aisways resistance should be determined in the sance period of panting respiration as are the other parameters, and this underlines the importance of ensuring fully automatic control on all selection operations.

Determinations made as described are read off on calibrated scales and it is desirable to have, 留 addition, a permanent record. Perhaps the most convenient way of achieving this is to use a mult channel recorder to which each channel of in formation can be fed. This method also permits the checking of the visually determined data. Sines small shifts of phase between channels would havie a large effect on parameters derived from the original information, it is necessary to minimize the shifts, whether arising in the recorder or the associated apparatus.

Direct photography of the pressure/volume and pressure/flow loops may be undertaken, but this requires simultaneous display on two oscilloscoses if the determinations are to be synchronous.

\section{REFERENCES}

Comroe, J. H., Botelho, S. Y., and DuBors, A. (1959): Design of a Body Plethysmograph for Studying Cardie pulmonary Physiology, f. appl. Physiol., 14, 439.

DuBois, A., Botelho, S. Y., Bedell, G. N., MARShall, R., and Comroe, J. H. (1956): A Rapid Plethysmograph Method for Measuring Thoracic Gas Volume, $\mathcal{~}$. clin. Invest., 35, 322.

, and ComroE, J. H. (r956): A New Method for Measuring Airway Resistance in Man Using a Bod Plethysmograph, Ibid., 35, 327.

Mead, J., and Whittengerger, J. L. W. (1953): Physical Properties of Human Lungs Measured During Spontaneous Respiration, Y. appl. Physiol., 8, 779.

MilleR, J. H., and Simmons, D. H. (I960): Rapid Determination of Dynamic Pulmonary Compliance and Resistance Ibid., 15, 967. 